\title{
Ações em saúde propostas em programas de transição do cuidado para idosos: revisão integrativa
}

\author{
Proposed health actions in care transition programs for older adults: an integrative review \\ Acciones de salud propuestas en los programas de transición del cuidado de los ancianos: revisión
}

integrativa

Recebido: 05/02/2022 | Revisado: 15/02/2022 | Aceito: 17/02/2022 | Publicado: 26/02/2022

Rodolfo Katalenic Petrocini

ORCID: https://orcid.org/0000-0002-4057-5728

Universidade de São Paulo, Brasil

E-mail: rodolfo_katalenic@usp.br

Marina Tomazela

ORCID: https://orcid.org/0000-0001-8416-2901

Universidade de São Paulo, Brasil

E-mail: marina.tomazela@usp.br

Gabriela Gonçalves Amaral

ORCID: https://orcid.org/0000-0002-9629-2815

Universidade de São Paulo, Brasil

E-mail: g.amaral@usp.br

Brener Santos Silva

ORCID: https://orcid.org/0000-0003-4610-3227

Universidade de São Paulo, Brasil

E-mail: brenersantos@usp.br

Ione Carvalho Pinto

ORCID: https://orcid.org/0000-0001-7541-5591

Universidade de São Paulo, Brasil

E-mail: ionecarv@eerp.usp.br

Fabiana Costa Machado Zacharias

ORCID: https://orcid.org/0000-0003-1150-6114

Universidade de São Paulo, Brasil

E-mail: fabiana.zacharias@usp.br

\begin{abstract}
Resumo
Este estudo visa analisar as evidências científicas acerca das ações em saúde propostas em Programas de Transição de Cuidados para idosos. O recorte escolhido para este estudo foi o de uma revisão integrativa. A estratégia de busca ocorreu no portal da Biblioteca Virtual de Saúde - que incorpora a LILACS, BDENF e IBECS; além da MEDLINE, SCOPUS, Embase e Web of Science, com descritores controlados e descritores não controlados (palavras-chave) que foram utilizados para a localização dos artigos. As buscas ocorreram no mês de janeiro de 2021. A amostra de artigos eleitos desta revisão integrativa foi composta por 37 artigos, sendo os anos de $2015(\mathrm{n}=4), 2016(\mathrm{n}=5), 2018(\mathrm{n}=$ 5), $2019(n=6)$ e $2020(n=5)$ os de maior número de publicações. Quanto às bases de dados, não foram encontrados artigos passíveis de serem eleitos no portal da BVS, que engloba a LILACS, IBECS e BDENF, em detrimento das outras bases em que se elegeu os artigos desta revisão, sendo elas: Scopus $(n=12)$, Embase $(n=5)$, Web Of Science $(\mathrm{n}=13)$, e Medline $(\mathrm{n}=7)$. Programas de Transição de Cuidados para idosos impactam diretamente na melhora da adesão à medicação, melhor estilo de vida saudável e melhores indicadores clínicos, bem como menores taxas de eventos adversos e readmissão hospitalares, por meio de ações em saúde como: educação do paciente e cuidador, ainda em ambiente hospitalar; acompanhamento telefônico e domiciliar após alta; supervisão e gestão de medicações e criação de planos terapêuticos individuais que visem a reabilitação nutricional, física e cognitiva.
\end{abstract}

Palavras-chave: Idoso; Alta hospitalar; Alta do paciente; Transição do cuidado.

\begin{abstract}
This study aimed to analyze the scientific evidence on health actions proposed in Transition of Care Programs for the elderly. The scope chosen for this study was an integrative review. The search strategy was carried out in the Virtual Health Library portal - which incorporates LILACS, BDENF and IBECS; as well as MEDLINE, SCOPUS, Embase and Web of Science, with controlled descriptors and non-controlled descriptors (key words) that were used to locate the articles. The searches occurred in the month of January 2021. The sample of articles elected for this integrative review was composed of 37 articles, with the years $2015(n=4), 2016(n=5), 2018(n=5), 2019(n=6)$ and $2020(n$ $=5$ ) being the years with the highest number of publications. As for the databases, no articles were found eligible in the VHL portal, which encompasses LILACS, IBECS and BDENF, to the detriment of the other bases in which the
\end{abstract}


articles of this review were chosen, being them: Scopus $(n=12)$, Embase $(n=5)$, Web Of Science $(n=13)$, and Medline $(n=7)$. Transitional care programs for the elderly directly impact on improved medication adherence, better healthy lifestyles, and better clinical indicators, as well as lower rates of adverse events and hospital readmission, through health actions such as: patient and caregiver education while still in the hospital environment; telephone and home follow-up after discharge; medication supervision and management; and creation of individual therapeutic plans aimed at nutritional, physical, and cognitive rehabilitation.

Keywords: Aged; Hospital discharge; Patient discharge; Care transition.

\section{Resumen}

Este estudio tuvo como objetivo analizar la evidencia científica sobre las acciones de salud propuestas en los Programas de Transición de Cuidados para personas mayores. El ámbito elegido para este estudio fue una revisión integradora. La estrategia de búsqueda se produjo en el portal de la Biblioteca Virtual de Salud - que incorpora LILACS, BDENF e IBECS; además de MEDLINE, SCOPUS, Embase y Web of Science, con descriptores controlados y descriptores no controlados (palabras clave) que se utilizaron para localizar los artículos. Los registros se produjeron en enero de 2021. La muestra de artículos elegidos de esta revisión integradora estuvo compuesta por 37 artículos, siendo los años $2015(n=4), 2016(n=5), 2018(n=5), 2019(n=6)$ y $2020(n=5)$ los años con mayor número de publicaciones. En cuanto a las bases de datos, no se encontraron artículos que pudieran ser elegidos en el portal de la BVS, que incluye LILACS, IBECS y BDENF, en detrimento de las otras bases en las que se eligieron los artículos de esta revisión, a saber: Scopus $(n=12)$, Embase $(n=5)$, Web Of Science $(n=13)$ y Medline $(n=7)$. Los programas de cuidados transitorios para ancianos tienen un impacto directo en la mejora de la adherencia a la medicación, un mejor estilo de vida saludable y mejores indicadores clínicos, así como menores tasas de eventos adversos y de reingreso hospitalario, a través de acciones sanitarias como: la educación del paciente y del cuidador mientras aún está en el entorno hospitalario; el seguimiento telefónico y domiciliario tras el alta; la supervisión y gestión de la medicación; y la creación de planes terapéuticos individuales dirigidos a la rehabilitación nutricional, física y cognitiva.

Palabras clave: Anciano; Alta hospitalaria; Alta del paciente; Transición de cuidado.

\section{Introdução}

Sabe-se que o processo de envelhecimento está relacionado à presença de comorbidades decorrentes de condições crônicas, como a hipertensão e diabetes, além de outras disfunções orgânicas que também contribuem para o aumento do acesso aos serviços de atenção à saúde.

Tendo em vista que os maiores custos dos sistemas de saúde são gerados no nível de atenção terciária, em função da maior complexidade tecnológica, segundo Rossetto et al..2019), os idosos com mais de 65 anos apresentam as maiores taxas de hospitalização, se comparados com as demais faixas etárias. Observa-se a necessidade em desenvolver ações e estratégias gerenciais, que promovam a integralidade do cuidado e melhores fluxos assistenciais em saúde, para essa parcela da população brasileira.

Nessa direção, pesquisadores vêm destinando esforços para melhor atender ao fenômeno de transição demográfica e seus desafios, frente ao envelhecimento populacional, de modo a assegurar qualidade assistencial por meio de ações terapêuticas eficazes, políticas públicas e melhor gestão de recursos e infraestrutura em saúde.

No que tange a estratégias para gestão de custos, em processos de hospitalização, uma proposta que vem se apresentando como promissora é a Transição de Cuidados, que consiste na etapa do processo assistencial em saúde de planejar e implementar ações que assegurem a continuidade do cuidado, na transferência de usuários pelos diferentes níveis de atenção à saúde (Registered Nurses' Association of Ontario, 2014). Trata-se de uma estratégia ainda pouco discutida no cenário mundial, mas que vem apresentando impacto na redução de custos com internações hospitalares e diminuição nas taxas de reinternações, ao pautar-se nos crescentes avanços tecnológicos para práticas terapêuticas de reabilitação (Flaming \& Haney, 2013).

Para tanto, a Transição de Cuidados é fundamentada em um plano abrangente de cuidados que inclui arranjos logísticos, educação do paciente e da família, e a coordenação entre os profissionais e gestores dos diferentes níveis de atenção à saúde (Coleman \& Boult, 2003; Burke et al., 2013).

Segundo Burke et al. (2013), a estrutura da Transição de Cuidados consiste em 10 domínios que necessitam ser 
contemplados, para que o processo ocorra de forma segura e efetiva, sendo eles: plano de alta; comunicação e informação abrangente sobre a doença e os cuidados; segurança de medicamentos; educação do paciente; promoção da autonomia; apoio social e comunitário; plano avançado de cuidado; coordenação do cuidado entre os membros da equipe de saúde; monitoramento e gerenciamento dos sintomas após a alta e acompanhamento nos serviços ambulatoriais.

De acordo com o parecer de Coleman et al., (2003), para que haja a efetivação de um Programa de Transição de Cuidados, este deve seguir quatro pilares essenciais, a saber: $1^{\circ}$ ) autogestão de medicação, em que os usuários precisam conhecer muito bem o esquema medicamentoso e contar com apoio e suporte dos profissionais de saúde, para auxiliá-los na reconciliação da lista de medicamentos pré e pós-hospitalização, identificando e corrigindo discrepâncias; $2^{\circ}$ ) registro dinâmico das condições de saúde, por meio do qual o usuário deve ser incentivado a registrar suas percepções, de todos os eventos que permeiam o processo de curso da doença, devendo os profissionais de saúde apoiar e incentivar os usuários a realizar e compartilhar os registros de saúde, a fim de favorecer a comunicação e transmissão de informações entre o usuário e a equipe de assistência pós-hospitalar; $3^{\circ}$ ) garantia do agendamento de consulta com médico especialista ou médico da Atenção Primária à Saúde, ainda no período de hospitalização, em que os profissionais precisam auxiliar os usuários no agendamento da próxima consulta, com o objetivo de garantir a continuidade do cuidado; e $4^{\circ}$ ) conhecimento, por parte do usuário, acerca dos sinais de piora ou mudanças no curso da doença, requerem orientações específicas da equipe multidisciplinar de saúde, para assegurar eficácia no processo de recuperação após hospitalização.

Por fim, entende-se que a Transição de Cuidados contempla um conjunto de ações coordenadas, destinadas a promover a continuidade assistencial, seja ela dada entre os diferentes níveis de atenção dos serviços de saúde, ou até mesmo de um serviço de saúde para os cuidados domiciliares (Coleman et al., 2003).

Diante disso, ao pensar na criação de fluxos assistenciais em saúde e na melhor gestão de custos hospitalares, devido ao crescente processo de transição demográfica no Brasil, é indicado investigar as evidências científicas acerca das ações em saúde. Analisar as propostas de programas de Transição de Cuidados, possibilita fomentar condutas para uma prática clínica, baseada em evidências que melhor atendam à população de idosos e suas demandas, pelos agravos de comorbidades e outras doenças debilitantes.

\section{Metodologia}

Trata-se de uma revisão integrativa que permite, a partir de uma temática específica, a reunião e síntese de estudos no vasto acervo da literatura científica, além de permitir gerar análises e conclusões acerca de determinado tema (Garcia et al., 2016).

Para tanto, foram adotadas as etapas estabelecidas por Mendes, Silveira e Galvão (2008), para a construção de uma revisão integrativa: 1) elaboração da pergunta da pesquisa, 2) seleção da amostragem dos estudos primários encontrados na literatura científica, 3) extração dos dados, 4) avaliação e síntese dos estudos primários incluídos, 5) análise e discussão dos resultados encontrados, e 6) apresentação da revisão.

No que se refere à formulação da pergunta da pesquisa, aplicou-se a estratégia PICo, um acrônimo da língua inglesa para Paciente, Intervenção e Contexto, que objetiva formular a questão de pesquisa e identificar as palavras-chave que irão auxiliar no processo de busca dos estudos primários (Kluwer, 2011). Assim, o elemento (P) consiste no idoso; o elemento (I) transição do cuidado e o elemento (Co), na hospitalização e alta hospitalar (Quadro 1). Logo, a questão de estudo definida para esta revisão de literatura foi: "Quais são as intervenções encontradas em programas hospitalares de transição de cuidado para idosos?" 
Quadro 1 - Aplicação da estratégia PICo.

\begin{tabular}{|c|c|c|}
\hline Acrônimo & Definição & Aplicação \\
\hline P & População & Idoso \\
\hline I & Intervenção & Transição do cuidado \\
\hline Co & Contexto & Hospitalização; Alta hospitalar \\
\hline
\end{tabular}

Fonte: Autores (2022).

A estratégia de busca ocorreu nas bases de dados do Portal Integrado da Biblioteca Virtual em Saúde (BVS) - que incorpora as bases de dados Latino-Americana e do Caribe em Ciência da Saúde (LILACS), Base de Dados de Enfermagem (BDENF) e Índice Bibliográfico Español en Ciencias de la Salud (IBECS); além da Medical Literature Analysis and Retrieval Sistem Online (MEDLINE), Scopus e Web of Science. As buscas ocorreram no mês de dezembro de 2021.

Os seguintes descritores controlados foram utilizados para pesquisa nas bases de dados, a partir dos Descritores em Ciências da Saúde (DeCS) da BVS; Medical Subject Headings (MeSH terms); e Emtree: Idoso; Idoso de 80 anos ou mais; Idoso Fragilizado e Cuidado Transicional. Os principais descritores não controlados (palavras-chave) selecionados foram: Programas de transição do cuidado e CTM-15. O cruzamento de descritores controlados e palavras-chave foi realizado com a utilização de Operadores Booleanos AND e OR.

Quadro 2 - Principais descritores controlados e descritores não controlados, de acordo com a estratégia PICo, conectados por operadores booleanos, para sintaxe nas bases de dados.

\begin{tabular}{|c|c|}
\hline Acrônimo & Descritores e palavras chaves \\
\hline P - População & Idoso OR “Idoso de 80 anos ou mais” OR “Idoso fragilizado" OR “Idoso debilitado" \\
\hline I - Intervenção & "Cuidado Transicional” OR “Transição de cuidado" OR "CTM 15” \\
\hline Co - Contexto & Hospitalização OR “Paciente Hospitalizado" OR "Alta Hospitalar” \\
\hline
\end{tabular}

Fonte: Autores (2022).

Para esta revisão foram definidos os seguintes critérios de inclusão: publicações encontradas nos idiomas em português, inglês e espanhol, sem limite de datas. Foram excluídos protocolos, normas técnicas, revisões de literatura, artigos com resumo e texto completo indisponíveis e estudos que não respondessem à questão de pesquisa proposta.

Após a busca de artigos nas bases de dados, utilizando as estratégias supracitadas, os estudos encontrados foram exportados para a ferramenta computacional denominada Rayyan, que dá suporte na organização e seleção dos artigos encontrados nas bases de dados. Por meio dessa ferramenta, são identificados artigos duplicados e classificados estudos primários, de acordo com os critérios de elegibilidade do estudo, que esquematiza de forma dinâmica o processo de tratamento da amostragem encontrada, para melhor gestão e visualização dos resultados (Ouzzani et al., 2016).

As leituras para seleção da amostra final foram realizadas por dois revisores, de forma independente, de modo que, quando houve discordância entre os artigos eleitos, um terceiro revisor foi convidado para a avaliação de elegibilidade.

A Figura 1 apresenta o fluxograma de seleção dos estudos primários encontrados nesta revisão integrativa. 
Figura 1 - Fluxo de seleção dos artigos da revisão integrativa.

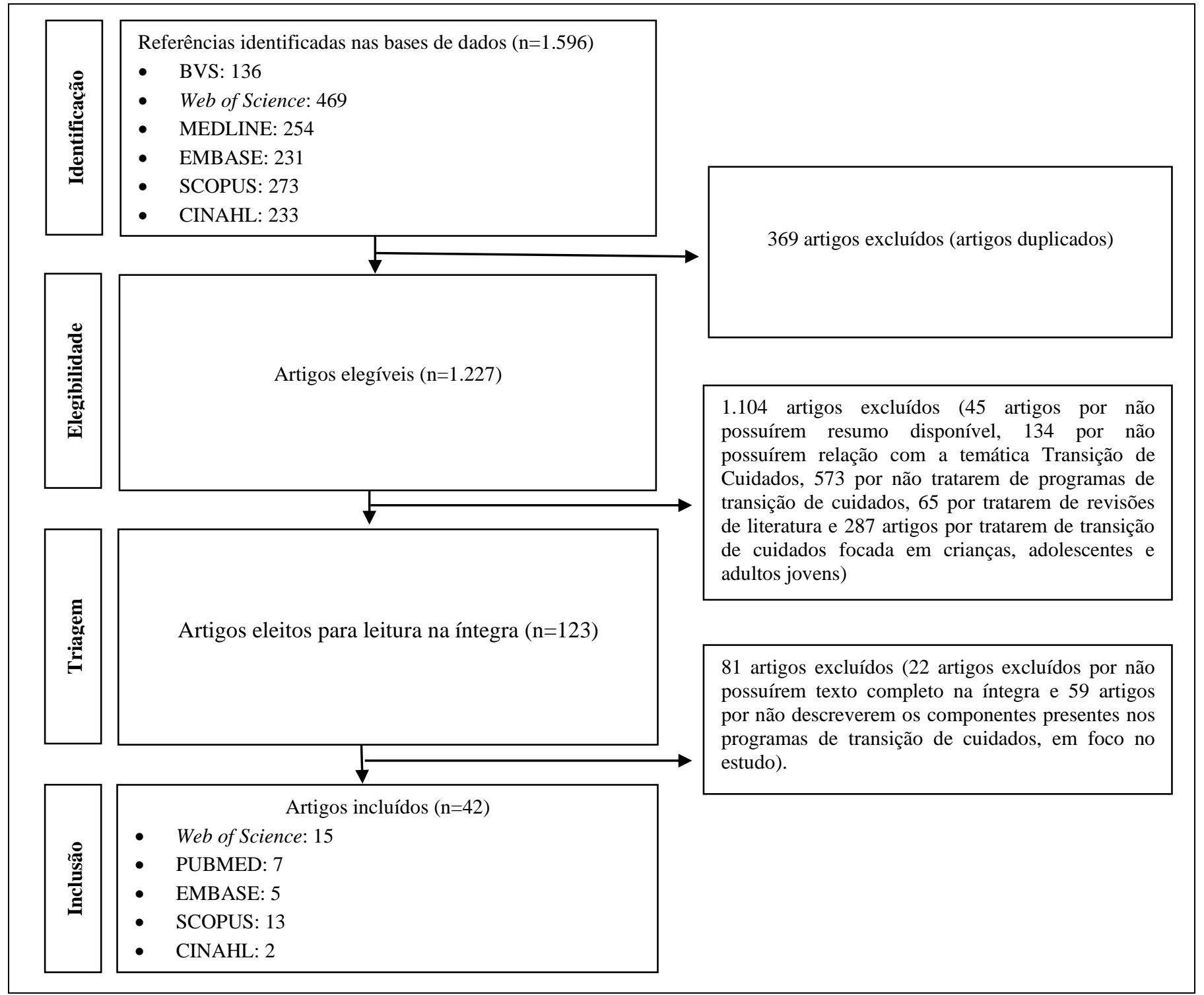

Fonte: Autores (2022).

Inicialmente, a amostra total de artigos encontrados nas bases de dados $(\mathrm{n}=1.596)$ foi exportada para o software Rayyan, o qual identificou, para respectiva exclusão, os artigos duplicados (n=369). Após a exclusão de duplicatas, realizou-se a avaliação da amostragem restante $(\mathrm{n}=1.227)$.

Na primeira análise, após leitura de título e resumo, obteve-se uma amostra de artigos eleitos para leitura na íntegra $(\mathrm{n}=123)$. No que tange às principais razões que excluíram, inicialmente, os estudos por não atenderem aos critérios de elegibilidade ( $n=1.104)$ estão: conflitos entre equipes de saúde na transferência de responsabilidade pós-internação do paciente; instrumentos de avaliação de cuidados transicionais; estudos com foco na transição da fase pediátrica para adulta e outros artigos que não atendiam à pergunta de pesquisa ou não possuíam relação com o tema do estudo.

$\mathrm{Na}$ segunda análise, após leitura dos artigos na íntegra, obteve-se uma amostra final concordante entre os dois revisores $(n=42)$, da qual justifica-se a exclusão dos outros artigos, posto que, embora abordassem programas de transição do cuidado, não tinham como foco discutir ou apresentar seus componentes estruturais, sem atender à pergunta do estudo. Outros 
artigos foram excluídos por não disponibilizarem texto completo e/ou gratuito, disponível para avaliação.

Para síntese dos resultados, um instrumento desenvolvido por pesquisadores da enfermagem foi utilizado, o qual descreve características pertencentes aos estudos eleitos na amostra desta revisão de literatura, permitindo sua identificação e melhor gestão da síntese. Dentre os componentes presentes no instrumento de coleta de informações destacam-se: metodologia aplicada no estudo, nível de evidência científica, objetivos e principais resultados (Ursi \& Galvão, 2006).

A classificação do nível de evidência dos estudos eleitos se deu por meio da classificação proposta pela "Prática baseada em evidências em enfermagem e saúde", sistematizada por Melnyk et al., (2001). Desta forma, a partir da metodologia descrita nos estudos, foi possível classificá-los em sete níveis, que são: nível 1 (mais forte), as evidências derivadas de revisão sistemática ou meta-análise de ensaios clínicos randomizados controlados; nível 2, as evidências resultantes de ensaios clínicos randomizados controlados bem delimitados; nível 3, evidências obtidas de ensaios clínicos bem delimitados sem randomização; nível 4, evidências obtidas de estudos de coorte e de caso-controle bem delimitados; nível 5, as evidências resultantes de revisão sistemática de estudos descritivos e qualitativos; nível 6, evidências originárias de um único estudo descritivo ou qualitativo e nível de evidência 7 (mais fraco), que abrange evidências obtidas a partir de opinião de autoridades/relatório de comitês de especialistas.

A análise dos resultados se deu de forma descritiva, apresentando a síntese dos estudos eleitos, assim como a comparação entre as semelhanças e diferenças, entre as propostas de ações observadas em programas de transição do cuidado.

\section{Resultados}

A amostra de artigos eleitos, nessa revisão integrativa, foi composta por 42 artigos, sendo que 2016 (n=5); 2018 $(n=5) ; 2019(n=6) ; 2020(n=5)$ e $2021(n=5)$ foram os anos com maior número de publicações. Quanto às bases de dados, não foram encontrados artigos passíveis de serem eleitos na LILACS, IBECS e BDENF, em detrimento das outras bases, Web Of Science $(\mathrm{n}=15)$, MEDLINE $(\mathrm{n}=7)$, EMBASE $(\mathrm{n}=5)$, SCOPUS $(\mathrm{n}=13)$ e CINAHL $(\mathrm{n}=2)$. Com relação ao idioma, todos os artigos eleitos encontravam-se na língua inglesa, não foi observado nenhum estudo proveniente de pesquisas realizadas no Brasil, o que sugere a escassez de estudos publicados sobre Programas de Transição de Cuidados em idosos no país. Na maioria dos casos, o enfermeiro foi tido como figura essencial para a efetivação de Programas de Transição de Cuidados (Quadro 3).

Quanto ao nível de evidência, 16 artigos eram constituídos por ensaios clínicos randomizados controlados e bem delimitados (nível de evidência II), 22 artigos apresentavam evidências obtidas de estudo de coorte bem delimitados (nível de evidência IV) e 4 artigos eram derivados de evidências originárias de estudos descritivos ou qualitativos (Quadro 3). 
Research, Society and Development, v. 11, n. 3, e35611326591, 2022

(CC BY 4.0) | ISSN 2525-3409 | DOI: htttp://dx.doi.org/10.33448/rsd-v11i3.26591

Quadro 3 - Identificação dos artigos eleitos (n=42).

\begin{tabular}{|c|c|c|c|c|c|}
\hline Artigo & Título/Autores/Ano/País & Método & $\begin{array}{c}\text { Nível de } \\
\text { evidência }\end{array}$ & Objetivo & Principais resultados \\
\hline E1 & $\begin{array}{l}\text { Título: Implementing a Heart Failure Transition Program to } \\
\text { Reduce } 30 \text {-Day Readmissions. } \\
\text { Autores: Hinch \& Staffileno. } \\
\text { Ano: } 2021 . \\
\text { País: Estados Unidos (EUA) }\end{array}$ & $\begin{array}{l}\text { Ensaio clínico } \\
\text { randomizado. }\end{array}$ & II & $\begin{array}{l}\text { Avaliar a influência de um Programa } \\
\text { de Transição de Cuidados na } \\
\text { redução das taxas de reinternações } \\
\text { de } 30 \text { dias por pacientes com } \\
\text { insuficiência cardíaca. }\end{array}$ & $\begin{array}{l}\text { Foi observada uma taxa de } \\
\text { readmissão hospitalar em } 30 \text { dias, } \\
\text { de } 18,2 \% \text {, sendo inferior à média } \\
\text { nacional de } 21,9 \% \text {. }\end{array}$ \\
\hline E2 & $\begin{array}{l}\text { Título: The nurse-coordinated cardiac care bridge } \\
\text { transitional care programme: a randomised clinical trial } \\
\text { Autores: Jepma et al. } \\
\text { Ano: } 2021 . \\
\text { País: Holanda }\end{array}$ & $\begin{array}{l}\text { Ensaio clínico } \\
\text { randomizado. }\end{array}$ & II & $\begin{array}{l}\text { Avaliar o impacto da combinação de } \\
\text { gerenciamento de casos, manejo de } \\
\text { doenças e reabilitação cardíaca } \\
\text { domiciliar na redução da readmissão } \\
\text { hospitalar e das taxas de } \\
\text { mortalidade. }\end{array}$ & $\begin{array}{l}\text { Este estudo não obteve sucesso em } \\
\text { sua implementação, em função da } \\
\text { dificuldade de manejar e monitorar } \\
\text { ações terapêuticas entre o grupo de } \\
\text { intervenção e controle. }\end{array}$ \\
\hline E3 & $\begin{array}{l}\text { Título: Feasibility and effectiveness of a multidimensional } \\
\text { post-discharge disease management programme for heart } \\
\text { failure patients in clinical practice: the HerzMobil Tirol } \\
\text { programme. } \\
\text { Autores: Poelzl et al. } \\
\text { Ano: } 2021 . \\
\text { País: Áustria }\end{array}$ & $\begin{array}{l}\text { Ensaio clínico não } \\
\text { randomizado }\end{array}$ & III & $\begin{array}{l}\text { Avaliar a viabilidade e eficácia de } \\
\text { um programa multidimensional, de } \\
\text { gerenciamento de doenças pós-alta, } \\
\text { usando um sistema de } \\
\text { monitoramento telemédico } \\
\text { incorporado a uma rede abrangente } \\
\text { de enfermeiros especialistas em } \\
\text { insuficiência cardíaca, médicos } \\
\text { residentes e centros de referência } \\
\text { secundários e terciários. }\end{array}$ & $\begin{array}{l}\text { O programa demonstrou eficácia na } \\
\text { redução das taxas de mortalidade } \\
\text { em } 6 \text { meses e } 1 \text { ano. }\end{array}$ \\
\hline E4 & $\begin{array}{l}\text { Título: Effects of a transitional care programme on } \\
\text { medication adherence in an older cardiac population: A } \\
\text { randomized clinical trial. } \\
\text { Autores: Daliri et al. } \\
\text { Ano: } 2021 . \\
\text { País: Amsterdam }\end{array}$ & $\begin{array}{l}\text { Ensaio clínico } \\
\text { randomizado. }\end{array}$ & II & $\begin{array}{l}\text { Avaliar o efeito de um Programa de } \\
\text { Transição de Cuidados voltado para } \\
\text { melhoria da adesão à medicação } \\
\text { pós-alta hospitalar. }\end{array}$ & $\begin{array}{l}\text { Com esse estudo foi possível } \\
\text { observar que um programa de } \\
\text { intervenção } \\
\text { multicomponentes, incluindo vários } \\
\text { componentes direcionados para } \\
\text { adesão à medicação em domicílio, } \\
\text { em pacientes cardíacos idosos que } \\
\text { receberam alta hospitalar, não } \\
\text { beneficiou seus níveis de adesão à } \\
\text { medicação. }\end{array}$ \\
\hline
\end{tabular}


Research, Society and Development, v. 11, n. 3, e35611326591, 2022

(CC BY 4.0) | ISSN 2525-3409 | DOI: htttp://dx.doi.org/10.33448/rsd-v11i3.26591

\begin{tabular}{|c|c|c|c|c|c|}
\hline E5 & $\begin{array}{l}\text { Título: Effects of a transitional care programme on } \\
\text { medication adherence in an older cardiac population: A } \\
\text { randomized clinical trial. } \\
\text { Autores: Gilbert et al. } \\
\text { Ano: } 2021 . \\
\text { País: França }\end{array}$ & $\begin{array}{l}\text { Ensaio clínico } \\
\text { randomizado. }\end{array}$ & II & $\begin{array}{l}\text { Avaliar o impacto de um Programa } \\
\text { de Transição de Cuidados liderado } \\
\text { por enfermeiros, sobre a taxa de } \\
\text { readmissões não programadas de } \\
\text { pacientes idosos, dentro de } 30 \text { dias } \\
\text { da alta das unidades de cuidados } \\
\text { agudos geriátricos. }\end{array}$ & $\begin{array}{l}\text { Por meio da intervenção, não foi } \\
\text { possível observar uma redução na } \\
\text { taxa de reinternações em } 30 \text { dias ou } \\
\text { visitas ao pronto-socorro de idosos } \\
\text { pacientes com risco de readmissão } \\
\text { hospitalar. }\end{array}$ \\
\hline E6 & $\begin{array}{l}\text { Título: Implementation of a Discharge Education Program to } \\
\text { Improve Transitions of Care for Patients at High Risk of } \\
\text { Medication Errors. } \\
\text { Autores: Crannage } \text { et al. } \\
\text { Ano: } 2020 . \\
\text { País: Estados Unidos (EUA) }\end{array}$ & \begin{tabular}{l}
\multicolumn{1}{c}{ Estudo } \\
observacional \\
prospectivo e \\
centralizado.
\end{tabular} & VI & $\begin{array}{l}\text { Desenvolver e avaliar um serviço de } \\
\text { educação de alta que une o ambiente } \\
\text { hospitalar e ambulatorial para } \\
\text { aumentar os pontos de contato do } \\
\text { paciente durante o processo de } \\
\text { Transição de Cuidados do hospital } \\
\text { para casa. }\end{array}$ & $\begin{array}{l}\text { A taxa de readmissão em } 30 \text { dias } \\
\text { foi de } 10 \% \text { para os pacientes que } \\
\text { receberam este serviço, em } \\
\text { comparação com } 19 \% \text {, antes da } \\
\text { implementação. }\end{array}$ \\
\hline E7 & $\begin{array}{l}\text { Título: Impact of Pharmacist Involvement in Heart Failure } \\
\text { Transition of Care. } \\
\text { Autores: Neu } \text { et al. } \\
\text { Ano: } 2020 . \\
\text { País: Estados Unidos (EUA) }\end{array}$ & $\begin{array}{l}\text { Estudo unicêntrico, } \\
\text { pré-pós quase- } \\
\text { experimental. }\end{array}$ & IV & $\begin{array}{l}\text { Avaliar o impacto na taxa de } \\
\text { readmissão hospitalar de um } \\
\text { programa de Transição de Cuidados } \\
\text { conduzido por farmacêuticos (TOC) } \\
\text { em pacientes com Insuficiência } \\
\text { Cardíaca (IC). }\end{array}$ & $\begin{array}{l}\text { O programa diminuiu, } \\
\text { significativamente, a taxa de } \\
\text { readmissão por IC, em } 30 \text { dias. No } \\
\text { grupo de controle, } 57 \text { (17,3\%) } \\
\text { pacientes tiveram readmissão por } \\
\text { IC em } 30 \text { dias, em comparação com } \\
35(10,5 \%) \text { pacientes no grupo de } \\
\text { intervenção. }\end{array}$ \\
\hline E8 & $\begin{array}{l}\text { Título: Evaluation of a Transitional Care Program After } \\
\text { Hospitalization for Heart Failure in an Integrated Health } \\
\text { Care System. } \\
\text { Autores: Baecker et al. } \\
\text { Ano: } 2020 . \\
\text { País: Estados Unidos (EUA) }\end{array}$ & $\begin{array}{l}\text { Análise } \\
\text { retrospectiva de } \\
\text { uma coorte clínica. }\end{array}$ & IV & $\begin{array}{l}\text { Investigar a relação entre estado } \\
\text { nutricional, capacidade funcional e } \\
\text { fragilidade em idosos participantes } \\
\text { de um programa de Assistência } \\
\text { Transicional de Idosos. }\end{array}$ & $\begin{array}{l}\text { Os pacientes que completaram o } \\
\text { Programa de Transição de } \\
\text { Cuidados, melhorias significativas } \\
\text { foram encontradas no estado } \\
\text { nutricional, fragilidade e } \\
\text { capacidade funcional. }\end{array}$ \\
\hline E9 & $\begin{array}{l}\text { Título: Effects of an integrated transitional care program for } \\
\text { stroke survivors living in a rural community: a randomized } \\
\text { controlled trial. } \\
\text { Autores: Deng et al. } \\
\text { Ano: } 2020 . \\
\text { País: China }\end{array}$ & $\begin{array}{l}\text { Ensaio clínico } \\
\text { randomizado. }\end{array}$ & II & $\begin{array}{l}\text { Avaliar os efeitos do manejo } \\
\text { transicional da saúde na adesão e } \\
\text { prognóstico em pacientes idosos } \\
\text { com infarto agudo do miocárdio, } \\
\text { submetidos à intervenção coronária } \\
\text { percutânea. }\end{array}$ & $\begin{array}{l}\text { Os pacientes participantes do } \\
\text { programa demonstraram melhor } \\
\text { adesão à medicação, adesão ao } \\
\text { reexame, estilo de vida saudável e } \\
\text { melhores indicadores clínicos, bem } \\
\text { como menores taxas de eventos } \\
\text { cardiovasculares adversos e } \\
\text { readmissão hospitalar. }\end{array}$ \\
\hline
\end{tabular}


Research, Society and Development, v. 11, n. 3, e35611326591, 2022

(CC BY 4.0) | ISSN 2525-3409 | DOI: htttp://dx.doi.org/10.33448/rsd-v11i3.26591

\begin{tabular}{|c|c|c|c|c|c|}
\hline E10 & $\begin{array}{l}\text { Título: Effectiveness of a health-social partnership program } \\
\text { for discharged non-frail older adults: a pilot study. } \\
\text { Autores: Wong et al. } \\
\text { Ano: } 2020 . \\
\text { País: China }\end{array}$ & $\begin{array}{l}\text { Ensaio clínico } \\
\text { randomizado. }\end{array}$ & II & $\begin{array}{l}\text { Avaliar se um programa iniciado em } \\
\text { um hospital que combinou Transição } \\
\text { de Cuidados e suporte de } \\
\text { autogerenciamento melhorou os } \\
\text { resultados a longo prazo para } \\
\text { pacientes hospitalizados devido à } \\
\text { Doença Pulmonar Obstrutiva } \\
\text { Crônica (DPOC). }\end{array}$ & $\begin{array}{l}\text { Este estudo não obteve êxito } \\
\text { significativo na redução de taxas de } \\
\text { retorno ao serviço de emergência } \\
\text { ou readmissão hospitalar. Sendo, } \\
\text { posteriormente, retratado pelos } \\
\text { autores. }\end{array}$ \\
\hline E11 & $\begin{array}{l}\text { Título: Evaluation of the impact of a post-hospital discharge } \\
\text { Transitional Aged Care Service on frailty, malnutrition and } \\
\text { functional ability. } \\
\text { Autores: Vearing et al. } \\
\text { Ano: } 2019 . \\
\text { País: Austrália }\end{array}$ & $\begin{array}{l}\text { Ensaio clínico } \\
\text { randomizado. }\end{array}$ & II & $\begin{array}{l}\text { Avaliar a eficácia comparativa das } \\
\text { intervenções de cuidados de } \\
\text { transição em readmissões } \\
\text { hospitalares não planejadas dentro } \\
\text { de } 28 \text { dias, } 12 \text { semanas e } 24 \text { semanas } \\
\text { após a alta hospitalar. }\end{array}$ & $\begin{array}{l}\text { Os participantes apresentaram } \\
\text { menor propensão a ter uma } \\
\text { readmissão não planejada, } 28 \text { dias } \\
\text { após a alta. Assim como uma } \\
\text { menor probabilidade de ter uma } \\
\text { readmissão não planejada nas } 12 \\
\text { semanas após alta. No entanto, não } \\
\text { houve diferenças significativas com } \\
\text { relação à comparação entre os } \\
\text { grupos de intervenção e controle, } \\
\text { após } 24 \text { semanas. }\end{array}$ \\
\hline E12 & $\begin{array}{l}\text { Título: Effects of transitional health management on } \\
\text { adherence and prognosis in elderly patients with acute } \\
\text { myocardial infarction in percutaneous coronary intervention: } \\
\text { A cluster randomized controlled trial. } \\
\text { Autores: Wu et al. } \\
\text { Ano: } 2019 . \\
\text { País: China }\end{array}$ & $\begin{array}{l}\text { Coorte } \\
\text { retrospectivo. }\end{array}$ & IV & $\begin{array}{l}\text { Avaliar a eficácia de um Programa } \\
\text { de Transição de Cuidados na } \\
\text { redução das taxas de readmissão em } \\
30 \text { dias. }\end{array}$ & $\begin{array}{l}\text { Os pacientes que receberam a } \\
\text { intervenção total do programa } \\
\text { tiveram } 86,6 \% \text { de redução nas } \\
\text { chances de readmissão, em } \\
\text { comparação com pacientes que não } \\
\text { receberam as intervenções. }\end{array}$ \\
\hline E13 & $\begin{array}{l}\text { Título: Excellence in Population Health: A Successful } \\
\text { Community-Based Care Transitions Program Model. } \\
\text { Autores: Warren et al. } \\
\text { Ano: } 2019 . \\
\text { País: Estados Unidos }\end{array}$ & $\begin{array}{l}\text { Estudo unicêntrico, } \\
\text { quase-experimental. }\end{array}$ & IV & $\begin{array}{l}\text { Estabelece um Programa de } \\
\text { Transição de Cuidados (TCP) com o } \\
\text { objetivo de diminuir o tempo de } \\
\text { internação, melhorar a eficiência da } \\
\text { alta e reduzir as reinternações de } \\
\text { pacientes neurocirúrgicos, } \\
\text { otimizando a educação do paciente e } \\
\text { vigilância pós-alta. }\end{array}$ & $\begin{array}{l}\text { O programa foi associado a um } \\
\text { tempo de espera, } \\
\text { significativamente, mais curto, alta } \\
\text { mais precoce e redução da } \\
\text { readmissão de } 30 \text { dias, após } \\
\text { neurocirurgia eletiva. }\end{array}$ \\
\hline E14 & $\begin{array}{l}\text { Título: Evaluation of a Multicomponent Care Transitions } \\
\text { Program for High-Risk Hospitalized Older Adults. } \\
\text { Autores: Huckfeldt et al. } \\
\text { Ano: } 2019 .\end{array}$ & $\begin{array}{l}\text { Ensaio clínico } \\
\text { randomizado. }\end{array}$ & II & $\begin{array}{l}\text { Avaliar se um modelo de enfermaria } \\
\text { virtual modificado, que incorpora } \\
\text { cuidados transitórios pré-alta } \\
\text { hospitalar pode reduzir readmissões }\end{array}$ & $\begin{array}{l}\text { Os pacientes do grupo intervenção } \\
\text { tiveram uma redução significativa } \\
\text { no número de readmissões em } 30 \\
\text { dias e no número de atendimentos }\end{array}$ \\
\hline
\end{tabular}


Research, Society and Development, v. 11, n. 3, e35611326591, 2022

(CC BY 4.0) | ISSN 2525-3409 | DOI: htttp://dx.doi.org/10.33448/rsd-v11i3.26591

\begin{tabular}{|c|c|c|c|c|c|}
\hline & País: Estados Unidos & & & $\begin{array}{l}\text { de pacientes com maior risco de } \\
\text { readmissão. }\end{array}$ & $\begin{array}{l}\text { no departamento de emergência em } \\
30 \text { dias, em comparação com } \\
\text { aqueles que não receberam a } \\
\text { intervenção. }\end{array}$ \\
\hline E15 & $\begin{array}{l}\text { Título: Implementation of a Cardiac Transitions of Care Pilot } \\
\text { Program: A Prospective Study of Inpatient and Outpatient } \\
\text { Clinical Pharmacy Services for Patients With Heart Failure } \\
\text { Exacerbation or Acute Myocardial Infarction. } \\
\text { Autores: Murphy et al. } \\
\text { Ano: } 2019 \text {. } \\
\text { País: Estados Unidos }\end{array}$ & $\begin{array}{c}\text { Estudo } \\
\text { observacional. }\end{array}$ & VI & $\begin{array}{l}\text { Avaliar se um programa piloto de } \\
\text { Transição de Cuidados diminui as } \\
\text { taxas de readmissão hospitalar para } \\
\text { pacientes com } 65 \text { anos ou mais, com } \\
\text { insuficiência cardíaca. }\end{array}$ & $\begin{array}{l}\text { O programa piloto reduziu as taxas } \\
\text { de readmissão, em } 30 \text { dias, em } \\
48 \% \text {; no entanto, observou-se que } \\
\text { os custos do programa foram bem } \\
\text { altos. }\end{array}$ \\
\hline E16 & $\begin{array}{l}\text { Título: Effect of a Hospital-Initiated Program Combining } \\
\text { Transitional Care and Long-term Self-management Support } \\
\text { on Outcomes of Patients Hospitalized With Chronic } \\
\text { Obstructive Pulmonary Disease: A Randomized Clinical } \\
\text { Trial. } \\
\text { Autores: Aboumatar et al. } \\
\text { Ano: } 2018 . \\
\text { País: Estados Unidos }\end{array}$ & $\begin{array}{l}\text { Ensaio clínico } \\
\text { randomizado. }\end{array}$ & II & $\begin{array}{l}\text { Examinar a eficácia de um programa } \\
\text { de cuidado transicional pautado na } \\
\text { prática avançada de enfermagem } \\
\text { para idosos hospitalizados com } \\
\text { insuficiência cardíaca. }\end{array}$ & $\begin{array}{l}\text { Em 52 semanas, os pacientes do } \\
\text { grupo de intervenção tiveram } \\
\text { menos readmissões e uma } \\
\text { diminuiçãa dos custos médios com } \\
\text { internação hospitalar. Ainda com o } \\
\text { grupo de intervenção, apenas a } \\
\text { curto prazo melhorias foram } \\
\text { demonstradas na qualidade de vida } \\
\text { geral e satisfação com o cuidado } \\
\text { recebido. }\end{array}$ \\
\hline E17 & $\begin{array}{l}\text { Título: Transitional care interventions reduce unplanned } \\
\text { hospital readmissions in highrisk older adults. } \\
\text { Autores: Finlayson et al. } \\
\text { Ano: } 2018 . \\
\text { País: Austrália }\end{array}$ & $\begin{array}{c}\text { Coorte } \\
\text { retrospectivo. }\end{array}$ & IV & $\begin{array}{l}\text { Verificar se um Programa de } \\
\text { Transição de Cuidados é capaz de } \\
\text { reduzir readmissões não planejadas } \\
\text { após uma internação médica aguda. }\end{array}$ & $\begin{array}{l}\text { Não houve mudança nas taxas de } \\
\text { readmissão ou atendimentos de } \\
\text { emergência. No entanto, nem todos } \\
\text { os pacientes receberam todos os } \\
\text { componentes da intervenção } \\
\text { propostos pelo programa. Esta } \\
\text { intervenção de transição não foi } \\
\text { bem-sucedida. }\end{array}$ \\
\hline E18 & $\begin{array}{l}\text { Título: Effect of Ambulatory Transitional Care Management } \\
\text { on 30-Day Readmission Rates. } \\
\text { Autores: Ballard et al. } \\
\text { Ano: } 2018 \text {. } \\
\text { País: Estados Unidos }\end{array}$ & $\begin{array}{l}\text { Ensaio clínico } \\
\text { randomizado. }\end{array}$ & II & $\begin{array}{l}\text { Avaliar a eficácia de um Programa } \\
\text { de Transição de Cuidados entre } \\
\text { pacientes com acidente vascular } \\
\text { cerebral. }\end{array}$ & $\begin{array}{l}\text { Os pacientes do grupo de } \\
\text { intervenção apresentaram melhoria } \\
\text { significativa na dimensão qualidade } \\
\text { de vida, bem como maior } \\
\text { satisfação, maiores escores do } \\
\text { Índice de Barthel Modificado e } \\
\text { menores escores de depressão, } \\
\text { quando comparados com o grupo } \\
\text { de controle. }\end{array}$ \\
\hline
\end{tabular}


Research, Society and Development, v. 11, n. 3, e35611326591, 2022

(CC BY 4.0) | ISSN 2525-3409 | DOI: htttp://dx.doi.org/10.33448/rsd-v11i3.26591

\begin{tabular}{|c|c|c|c|c|c|}
\hline E19 & $\begin{array}{l}\text { Título: Transitional care services: a quality and safety } \\
\text { process improvement program in neurosurgery. } \\
\text { Autores: Robertson et al. } \\
\text { Ano: } 2018 \text {. } \\
\text { País: Estados Unidos }\end{array}$ & $\begin{array}{l}\text { Coorte } \\
\text { retrospectivo. }\end{array}$ & IV & $\begin{array}{l}\text { Examinar a associação entre as } \\
\text { intervenções de Transição de } \\
\text { Cuidados e reinternações por todas } \\
\text { as causas, em } 30 \text { dias após alta } \\
\text { hospitalar. }\end{array}$ & $\begin{array}{l}\text { Tanto a exposição a uma visita } \\
\text { domiciliar de saúde, dentro de } 2 \\
\text { dias após a alta, quanto uma } \\
\text { chamada de telefone por um } \\
\text { especialista, estiveram associados a } \\
\text { uma menor taxa de readmissão } \\
\text { hospitalar. Uma visita clínica de } 7 \\
\text { dias foi associada a uma taxa de } \\
\text { readmissão mais baixa em } \\
\text { comparação com nenhuma visita } \\
\text { clínica. }\end{array}$ \\
\hline E20 & $\begin{array}{l}\text { Título: RETRACTED: Effect of a Program Combining } \\
\text { Transitional Care and Long-term Self-management Support } \\
\text { on Outcomes of Hospitalized Patients With Chronic } \\
\text { Obstructive Pulmonary Disease A Randomized Clinical Trial } \\
\text { (Retracted article. See vol. 322, pg. 1417, 2019). } \\
\text { Autores: Hanan et al. } \\
\text { Ano: } 2018 . \\
\text { País: Estados Unidos }\end{array}$ & $\begin{array}{l}\text { Estudo quase } \\
\text { experimental. }\end{array}$ & IV & $\begin{array}{l}\text { Avaliar se um programa de cuidados } \\
\text { domiciliares de transição operado } \\
\text { por um hospital foi eficaz na } \\
\text { redução de reinternações. }\end{array}$ & $\begin{array}{l}\text { Os pacientes inscritos no programa } \\
\text { de cuidados domiciliares de } \\
\text { transição tiveram uma utilização } \\
\text { hospitalar aguda, } \\
\text { significativamente, menor por meio } \\
\text { da redução de atendimentos em } \\
\text { prontos-socorros e internações } \\
\text { hospitalares. }\end{array}$ \\
\hline E21 & $\begin{array}{l}\text { Título: Performance Improvement to Decrease Readmission } \\
\text { Rates for Patients With a Left Ventricular Assist Device. } \\
\text { Autores: Iseler } \text { et al. } \\
\text { Ano: } 2018 . \\
\text { País: Estados Unidos }\end{array}$ & $\begin{array}{l}\text { Estudo descritivo } \\
\text { retrospectivo }\end{array}$ & VI & $\begin{array}{l}\text { Avaliar a eficácia de um Programa } \\
\text { de Transição de Cuidados. }\end{array}$ & $\begin{array}{l}\text { O programa gerou maior nível de } \\
\text { satisfação do paciente com o } \\
\text { processo de transição de cuidados, } \\
\text { além de melhorar a confiança dos } \\
\text { participantes com o autocuidado. } \\
\text { Além disso, o programa } \\
\text { demonstrou uma redução de } 73 \% \\
\text { nas readmissões e uma economia } \\
\text { real de custos durante seu período } \\
\text { de } 9 \text { meses do estudo. }\end{array}$ \\
\hline E22 & $\begin{array}{l}\text { Título: Applying the Integrated Practice Unit Concept to a } \\
\text { Modified Virtual Ward Model of Care for Patients at Highest } \\
\text { Risk of Readmission: A Randomized Controlled Trial. } \\
\text { Autores: Low et al. } \\
\text { Ano: 2017. } \\
\text { País: Singapura }\end{array}$ & $\begin{array}{l}\text { Ensaio clínico } \\
\text { randomizado. }\end{array}$ & II & $\begin{array}{l}\text { Avaliar o impacto de um programa } \\
\text { de Transição de Cuidados na taxa de } \\
\text { reinternações hospitalar. }\end{array}$ & $\begin{array}{l}\text { Os pacientes que receberam a } \\
\text { intervenção experimentaram um } \\
\text { terço a menos de reinternações do } \\
\text { que aqueles pacientes do grupo } \\
\text { comparação, produzindo uma } \\
\text { economia de custos. }\end{array}$ \\
\hline
\end{tabular}


Research, Society and Development, v. 11, n. 3, e35611326591, 2022

(CC BY 4.0) | ISSN 2525-3409 | DOI: htttp://dx.doi.org/10.33448/rsd-v11i3.26591

\begin{tabular}{|c|c|c|c|c|c|}
\hline E23 & $\begin{array}{l}\text { Título: Short termand long term effective nessofapost hospital } \\
\text { care transitions program in an older, medically complex } \\
\text { population. } \\
\text { Autores: Takahashi et al. } \\
\text { Ano: } 2016 . \\
\text { País: Estados Unidos }\end{array}$ & Caso-controle. & IV & $\begin{array}{l}\text { Avaliar a eficácia de um Programa } \\
\text { de Transição na redução das taxas de } \\
\text { reinternação e/ou mortalidade, após } \\
\text { alta hospitalar. }\end{array}$ & $\begin{array}{l}\text { O programa reduziu a taxa de } \\
\text { readmissão de } 30 \text { dias, para o grupo } \\
\text { de estudo, em } 15 \% \text {, embora a taxa } \\
\text { esperada fosse de } 20 \% \text {. A taxa de } \\
\text { mortalidade observada foi de } 2 \% \text {, } \\
\text { para o grupo de estudo, com uma } \\
\text { taxa esperada de } 7 \% \text {. }\end{array}$ \\
\hline E24 & $\begin{array}{l}\text { Título: The development, implementation and evaluation of a } \\
\text { transitional care programme to improve outcomes of frail } \\
\text { older patients after hospitalisation. } \\
\text { Autores: Heim et al. } \\
\text { Ano: } 2016 . \\
\text { País: Estados Unidos }\end{array}$ & $\begin{array}{c}\text { Coorte } \\
\text { retrospectivo. }\end{array}$ & IV & $\begin{array}{l}\text { Definir um amplo espectro de } \\
\text { intervenções e serviços para } \\
\text { pacientes idosos com alto risco de } \\
\text { readmissão hospitalar. }\end{array}$ & $\begin{array}{l}\text { Após implementação de um } \\
\text { modelo de Programa de Transição } \\
\text { de Cuidados, a taxa de readmissão } \\
\text { hospitalar diminuiu para } 12,5 \% \text {. }\end{array}$ \\
\hline E25 & $\begin{array}{l}\text { Título: Effects of a transitional palliative care model on } \\
\text { patients with end-stage heart failure: a randomised controlled } \\
\text { trial. } \\
\text { Autores: Wong et al. } \\
\text { Ano: } 2016 . \\
\text { País: China }\end{array}$ & $\begin{array}{l}\text { Ensaio clínico } \\
\text { randomizado. }\end{array}$ & II & $\begin{array}{l}\text { Avaliar um modelo de Programa de } \\
\text { Transição de Cuidados iniciado em } \\
\text { um hospital que combinava suporte } \\
\text { de transição e autocuidado, de longo } \\
\text { prazo, para pacientes hospitalizados } \\
\text { devido à Doença Pulmonar } \\
\text { Obstrutiva Crônica (DPOC) e seus } \\
\text { cuidadores e/ou familiares. }\end{array}$ & $\begin{array}{l}\text { O programa, com duração de } 3 \\
\text { meses, que combinou transição e } \\
\text { suporte de autogerenciamento de } \\
\text { longo prazo, resultou, } \\
\text { significativamente, na redução de } \\
\text { hospitalizações relacionadas à } \\
\text { DPOC e visitas ao departamento de } \\
\text { emergência, bem como melhorou a } \\
\text { qualidade de vida relacionada à } \\
\text { saúde, } 6 \text { meses após alta hospitalar. }\end{array}$ \\
\hline E26 & $\begin{array}{l}\text { Título: Reducing Preventable Hospitalizations With Two } \\
\text { Models of Transitional Care. } \\
\text { Autores: Morrison et al. } \\
\text { Ano: } 2016 . \\
\text { País: Estados Unidos }\end{array}$ & Coorte prospectivo. & IV & $\begin{array}{l}\text { Comparar a utilização de curto prazo } \\
\text { ( } 30 \text { dias) e de longo prazo ( } 180 \text { dias) } \\
\text { de participantes inscritos em um } \\
\text { modelo de Programa de Transição } \\
\text { de Cuidados. }\end{array}$ & $\begin{array}{l}\text { Foi observado uma redução, nas } \\
\text { taxas de reinternação, em } 30 \text { dias, } \\
\text { entre aqueles inscritos no } \\
\text { programa, em comparação com os } \\
\text { referentes. No entanto, este efeito } \\
\text { não foi observado em } 180 \text { dias. }\end{array}$ \\
\hline E27 & $\begin{array}{l}\text { Título: A Patient-Centered Transitional Care Case } \\
\text { Management Program: Taking Case Management to the } \\
\text { Streets and Beyond. } \\
\text { Autores: Lovelace } \text { et al. } \\
\text { Ano: } 2016 . \\
\text { País: Estados Unidos }\end{array}$ & $\begin{array}{l}\text { Coorte } \\
\text { retrospectivo. }\end{array}$ & IV & $\begin{array}{l}\text { Avaliar a eficácia de um protocolo } \\
\text { de cuidados na redução das taxas de } \\
\text { readmissão hospitalar. }\end{array}$ & $\begin{array}{l}\text { Os participantes do programa } \\
\text { apresentaram uma redução de } \\
44,3 \% \text { nas taxas de readmissão, em } \\
30 \text { dias, em comparação com as } \\
\text { taxas médias de readmissão, em } 30 \\
\text { dias do hospital. }\end{array}$ \\
\hline
\end{tabular}


Research, Society and Development, v. 11, n. 3, e35611326591, 2022

(CC BY 4.0) | ISSN 2525-3409 | DOI: htttp://dx.doi.org/10.33448/rsd-v11i3.26591

\begin{tabular}{|c|c|c|c|c|c|}
\hline E28 & $\begin{array}{l}\text { Título: Evaluation of a New Zealand program to improve } \\
\text { transition of care for older high risk adults. } \\
\text { Autores: Zhou et al. } \\
\text { Ano: } 2015 \text {. } \\
\text { País: Nova Zelândia }\end{array}$ & Coorte prospectivo. & IV & $\begin{array}{l}\text { Comparar o risco de reinternações } \\
\text { não planejadas de indivíduos } \\
\text { participantes de um modelo de } \\
\text { Programa de Transição de Cuidados. }\end{array}$ & $\begin{array}{l}\text { Os participantes do programa } \\
\text { tiveram menos reinternações não } \\
\text { planejadas e menores visitas ao } \\
\text { pronto-socorro, após a alta } \\
\text { hospitalar. }\end{array}$ \\
\hline E29 & $\begin{array}{l}\text { Título: Effectiveness of a transitional home care program in } \\
\text { reducing acute hospital utilization: A quasi-experimental } \\
\text { study. } \\
\text { Autores: Low et al. } \\
\text { Ano: } 2015 . \\
\text { País: Singapura }\end{array}$ & $\begin{array}{c}\text { Caso-controle } \\
\text { quase experimental }\end{array}$ & IV & $\begin{array}{l}\text { Investigar o impacto de um } \\
\text { programa de melhoria da qualidade e } \\
\text { cuidados integrados de transição } \\
\text { pós-alta em um sistema hospitalar. }\end{array}$ & $\begin{array}{l}\text { Os pacientes que receberam } \\
\text { intervenções tiveram taxas mais } \\
\text { baixas de readmissão e morte do } \\
\text { que o grupo controle, dentro de } 30 \\
\text { dias após a alta. }\end{array}$ \\
\hline E30 & $\begin{array}{l}\text { Título: Effects of a 4-week transitional care programme for } \\
\text { discharged stroke survivors in Hong Kong: a randomised } \\
\text { controlled trial. } \\
\text { Autores: Wong et al. } \\
\text { Ano: } 2015 . \\
\text { País: China }\end{array}$ & $\begin{array}{l}\text { Ensaio Clínico } \\
\text { Randomizado }\end{array}$ & II & $\begin{array}{l}\text { Avaliar se um modelo de Programa } \\
\text { de Transição de Cuidados pode } \\
\text { reduzir as taxas de readmissão de } \\
\text { pacientes com insuficiência } \\
\text { cardíaca. }\end{array}$ & $\begin{array}{l}\text { A intervenção reduziu em } 48 \% \text { as } \\
\text { taxas de readmissão, em } 30 \text { dias do } \\
\text { hospital durante o período pós- } \\
\text { intervenção. No entanto, a } \\
\text { intervenção teve pouco efeito sobre } \\
\text { o tempo de internação ou os custos } \\
\text { diretos totais em } 60 \text { dias. }\end{array}$ \\
\hline E31 & $\begin{array}{l}\text { Título: Protocol-Driven Allied Health Post-Discharge } \\
\text { Transition Clinic to Reduce Hospital Readmissions in Heart } \\
\text { Failure. } \\
\text { Autores: Donaho et al. } \\
\text { Ano: } 2015 . \\
\text { País: Estados Unidos }\end{array}$ & $\begin{array}{c}\text { Caso-controle } \\
\text { quase experimental }\end{array}$ & IV & $\begin{array}{l}\text { Verificar a eficácia de uma } \\
\text { intervenção proposta em um } \\
\text { Programa de Transição de Cuidados } \\
\text { multicomponente direcionado a } \\
\text { pacientes hospitalizados, com } 75 \\
\text { anos ou mais, e com alto risco de } \\
\text { readmissões hospitalares. }\end{array}$ & $\begin{array}{l}\text { Esta intervenção não foi eficaz na } \\
\text { redução de reinternações de } 30 \text { ou } \\
7 \text { dias, tampouco quanto à taxa de } \\
\text { visitas de retorno ao serviço de } \\
\text { emergência. }\end{array}$ \\
\hline E32 & $\begin{array}{l}\text { Título: Transitional Care Models Preventing Readmissions } \\
\text { for High-Risk Patient Populations. } \\
\text { Autores: Mae et al. } \\
\text { Ano: } 2014 . \\
\text { País: Estados Unidos }\end{array}$ & Coorte Prospectivo & IV & $\begin{array}{l}\text { Determinar a mudança nas taxas de } \\
\text { readmissão em } 30 \text { dias para } \\
\text { pacientes com exacerbação de IC ou } \\
\text { IAM após a implementação de um } \\
\text { padrão de tratamento previstos em } \\
\text { um Programa de Transição de } \\
\text { Cuidados. }\end{array}$ & $\begin{array}{l}\text { As taxas de readmissão em } 30 \text { dias } \\
\text { não foram, estatisticamente, } \\
\text { significativas quando comparadas } \\
\text { às taxas de readmissão } \\
\text { institucionais históricas, antes do } \\
\text { início do estudo. }\end{array}$ \\
\hline E33 & $\begin{array}{l}\text { Título: Effectiveness of a National Transitional Care } \\
\text { Program in Reducing Acute Care Use. } \\
\text { Autores: Wee et al. } \\
\text { Ano: } 2014 . \\
\text { País: Singapura }\end{array}$ & $\begin{array}{c}\text { Caso-controle } \\
\text { quase experimental }\end{array}$ & IV & $\begin{array}{l}\text { Relatar o desenvolvimento, } \\
\text { implementação e avaliação de um } \\
\text { programa regional de cuidados de } \\
\text { transição, visando melhorar a taxa } \\
\text { de recuperação de pacientes idosos } \\
\text { frágeis. }\end{array}$ & $\begin{array}{l}\text { Em pacientes frágeis, a incidência } \\
\text { de eventos adversos diminuiu de } \\
49,2 \% \text {, na amostra pré-programa, } \\
\text { para } 35,5 \% \text {, na amostra pós- } \\
\text { programa. }\end{array}$ \\
\hline
\end{tabular}


Research, Society and Development, v. 11, n. 3, e35611326591, 2022

(CC BY 4.0) | ISSN 2525-3409 | DOI: htttp://dx.doi.org/10.33448/rsd-v11i3.26591

\begin{tabular}{|c|c|c|c|c|c|}
\hline E34 & $\begin{array}{l}\text { Título: Transitional care for patients with chronic obstructive } \\
\text { pulmonary disease. } \\
\text { Autores: Cheng et al. } \\
\text { Ano: } 2014 \text {. } \\
\text { País: Singapura }\end{array}$ & $\begin{array}{c}\text { Caso-controle } \\
\text { quase experimental }\end{array}$ & IV & $\begin{array}{l}\text { Observar os efeitos de cuidados de } \\
\text { transição na qualidade de vida de } \\
\text { pacientes com Doença Pulmonar } \\
\text { Obstrutiva Crônica (DPOC). }\end{array}$ & $\begin{array}{l}\text { O programa melhorou a qualidade } \\
\text { de vida relacionada à saúde em } \\
\text { pacientes com DPOC que sofreram } \\
\text { recentemente uma agudização. }\end{array}$ \\
\hline E35 & $\begin{array}{l}\text { Título: Evaluation of a modified community based care } \\
\text { transitions model to reduce costs and improve outcomes. } \\
\text { Autores: Logue } \text { et al. } \\
\text { Ano: } 2013 \text {. } \\
\text { País: Estados Unidos }\end{array}$ & $\begin{array}{l}\text { Coorte prospectivo } \\
\text { quase experimental }\end{array}$ & IV & $\begin{array}{l}\text { Avaliar o impacto da adaptação de } \\
\text { um modelo de Programa de } \\
\text { Transição de Cuidados com o } \\
\text { objetivo de atender às necessidades } \\
\text { de idosos com doenças crônicas, } \\
\text { durante episódios agudos de doença. }\end{array}$ & $\begin{array}{l}\text { Os resultados demonstram que um } \\
\text { modelo rigorosamente testado de } \\
\text { Transição de Cuidados para idosos } \\
\text { com doenças crônicas pode ser } \\
\text { adaptado com sucesso. } \\
\text { Demonstrando, principalmente, } \\
\text { redução de custos para o serviço de } \\
\text { saúde. }\end{array}$ \\
\hline E36 & $\begin{array}{l}\text { Título: High-value transitional care: Translation of research } \\
\text { into practice. } \\
\text { Autores: Naylor et al. } \\
\text { Ano: } 2013 \text {. } \\
\text { País: Estados Unidos }\end{array}$ & $\begin{array}{l}\text { Ensaio clínico } \\
\text { randomizado }\end{array}$ & II & $\begin{array}{l}\text { Examinar os efeitos de cuidados de } \\
\text { transição em pacientes paliativos } \\
\text { com insuficiência cardíaca em } \\
\text { estágio terminal, após a alta } \\
\text { hospitalar. }\end{array}$ & $\begin{array}{l}\text { O grupo de intervenção teve uma } \\
\text { taxa de readmissão hospitalar } \\
\text { significativamente menor }(33,6 \%) \\
\text { do que o grupo de controle } \\
(61,0 \%) \text {, em } 12 \text { semanas. }\end{array}$ \\
\hline E37 & $\begin{array}{l}\text { Título: Low-cost transitional care with nurse managers } \\
\text { making mostly phone contact with patients cut } \\
\text { rehospitalization at A VA hospital. } \\
\text { Autores: Kind et al. } \\
\text { Ano: } 2012 \text {. } \\
\text { País: Estados Unidos }\end{array}$ & $\begin{array}{l}\text { Ensaio clínico } \\
\text { randomizado }\end{array}$ & II & $\begin{array}{l}\text { Avaliar a eficácia de um Programa } \\
\text { de Transição de Cuidados para } \\
\text { idosos. }\end{array}$ & $\begin{array}{l}\text { Não houve diferenças significativas } \\
\text { no grupo intervenção em } \\
\text { comparação com o grupo controle. }\end{array}$ \\
\hline E38 & $\begin{array}{l}\text { Título: Integrated postdischarge transitional care in a } \\
\text { hospitalist system to improve discharge outcome: an } \\
\text { experimental study. } \\
\text { Autores: Shu et al. } \\
\text { Ano: } 2011 . \\
\text { País: China }\end{array}$ & $\begin{array}{l}\text { Ensaio clínico } \\
\text { randomizado }\end{array}$ & II & $\begin{array}{l}\text { Avaliar o efeito de um programa } \\
\text { integrado de Transição de Cuidados } \\
\text { sobre os resultados de indicadores } \\
\text { de saúde, em sobreviventes de AVC. }\end{array}$ & $\begin{array}{l}\text { Houve melhora significativa dos } \\
\text { indicadores físicos e mentais dos } \\
\text { pacientes que estiveram no grupo } \\
\text { intervenção. }\end{array}$ \\
\hline E39 & $\begin{array}{l}\text { Título: Effectiveness and Cost of a Transitional Care } \\
\text { Program for Heart Failure A Prospective Study With } \\
\text { Concurrent Controls. } \\
\text { Autores: Stauffer } \text { et al. } \\
\text { Ano: } 2011 \text {. } \\
\text { País: Estados Unidos }\end{array}$ & $\begin{array}{c}\text { Coorte } \\
\text { Retrospectivo }\end{array}$ & IV & $\begin{array}{l}\text { Descrever as abordagens e os } \\
\text { resultados de dois programas de } \\
\text { cuidados de transição distintos que } \\
\text { atendem a diferentes populações: um } \\
\text { é fornecido por especialistas em } \\
\text { enfermagem clínica com foco no } \\
\text { autogerenciamento de doenças } \\
\text { crônicas, outro por médicos }\end{array}$ & $\begin{array}{l}\text { Ambos os Programas de Transição } \\
\text { de Cuidados apresentaram redução } \\
\text { significativa das taxas de } \\
\text { reinternações hospitalares. }\end{array}$ \\
\hline
\end{tabular}


Research, Society and Development, v. 11, n. 3, e35611326591, 2022

(CC BY 4.0) | ISSN 2525-3409 | DOI: htttp://dx.doi.org/10.33448/rsd-v11i3.26591

\begin{tabular}{|c|c|c|c|c|c|}
\hline & & & & $\begin{array}{l}\text { especializados } \\
\text { paliativos. }\end{array}$ & \\
\hline E40 & $\begin{array}{l}\text { Título: To the hospital and back home again: a nurse } \\
\text { practitioner-based transitional care program for hospitalized } \\
\text { homebound people. } \\
\text { Autores: Ornstein } \text { et al. } \\
\text { Ano: } 2011 \text {. } \\
\text { País: Estados Unidos }\end{array}$ & $\begin{array}{c}\text { Coorte } \\
\text { Retrospectivo }\end{array}$ & IV & $\begin{array}{l}\text { Examinar o impacto de um modelo } \\
\text { de Programa de Transição de } \\
\text { Cuidados na redução de admissões } \\
\text { hospitalares. }\end{array}$ & $\begin{array}{l}\text { Os pacientes que obtiveram a } \\
\text { intervenção, nos anos fiscais de } \\
2013 \text { e } 2014 \text {, experimentaram uma } \\
\text { redução de } 67 \% \text { nas admissões } \\
\text { hospitalares e uma redução de } 61 \% \\
\text { nas visitas ao pronto-socorro nos } \\
90 \text { dias após a participação deste } \\
\text { programa. }\end{array}$ \\
\hline E41 & $\begin{array}{l}\text { Título: A hybrid transitional care program. } \\
\text { Autores: Daley et al. } \\
\text { Ano: } 2010 . \\
\text { País: Estados Unidos }\end{array}$ & Estudo descritivo & IV & $\begin{array}{l}\text { Avaliar a eficácia e viabilidade de } \\
\text { um modelo de transição de cuidado } \\
\text { para o atendimento de pacientes com } \\
\text { dispositivos ventriculares esquerdos. }\end{array}$ & $\begin{array}{l}\text { A taxa de readmissão de } 30 \text { dias, } \\
\text { durante o piloto do estudo, foi de } \\
14,3 \% \text {, em comparação com a taxa } \\
\text { geral anual anterior de } 42,6 \% \text {. }\end{array}$ \\
\hline E42 & $\begin{array}{l}\text { Título: Transitional Care of Older Adults Hospitalized with } \\
\text { Heart Failure: A Randomized, Controlled Trial. } \\
\text { Autores: Naylor et al. } \\
\text { Ano: } 2004 . \\
\text { País: Estados Unidos }\end{array}$ & Estudo descritivo & IV & $\begin{array}{l}\text { Avaliar a eficácia de um programa } \\
\text { piloto de Transição de Cuidados } \\
\text { projetado para melhorar a } \\
\text { coordenação e a continuidade dos } \\
\text { cuidados, a fim de reduzir as } \\
\text { readmissões hospitalares. }\end{array}$ & $\begin{array}{l}\text { A intervenção não foi efetiva para } \\
\text { redução do tempo de internação } \\
\text { hospitalar e da taxa de readmissão } \\
\text { hospitalar, para as pessoas que } \\
\text { foram hospitalizadas e receberam } \\
\text { alta. }\end{array}$ \\
\hline
\end{tabular}

Fonte: Autores (2022). 
Research, Society and Development, v. 11, n. 3, e35611326591, 2022

(CC BY 4.0) | ISSN 2525-3409 | DOI: http://dx.doi.org/10.33448/rsd-v11i3.26591

Os estudos propostos para relatar os programas de Transição de Cuidados foram conduzidos, predominantemente, em pacientes idosos, acima de 60 anos, que ainda se encontravam hospitalizados e prestes a receber alta hospitalar para domicílio. Muitos dos estudos eleitos possuem foco em pacientes que foram hospitalizados por apresentar agudização de comorbidades associadas a doenças crônicas não transmissíveis $(\mathrm{n}=17)$.

Tendo em vista as evidências do tipo II e IV encontradas, sobre a utilização de Programas de Transição de Cuidados na reabilitação de pacientes que receberam alta hospitalar, reduzindo as taxas de readmissão e melhorando a qualidade de vida após internação, pode-se constatar a eficácia da proposta de Transição de Cuidados. As principais ações terapêuticas observadas foram: educação do paciente e cuidador, ainda em ambiente hospitalar; acompanhamento telefônico e domiciliar após alta; supervisão de medicações e planos terapêuticos individuais que visam a reabilitação nutricional, física e cognitiva.

\section{Discussão}

Para amparo ao idoso, os Programas de Transição de Cuidados se apresentam como ferramentas que devem ser usadas para assegurar a assistência em saúde de qualidade, integralizada e continuada no processo de transferência dos pacientes entre os diferentes pontos da rede de atenção à saúde.

Segundo Finlayson et al. (2018), para atender às exigências do idoso, é necessário que haja dedicação de todos os profissionais envolvidos no âmbito assistencial, de modo a utilizar as tecnologias disponíveis, a fim de aprimorar condutas terapêuticas, focadas em singularidades no sentido de promover a autonomia, a reabilitação física e cognitiva dessa parcela da população.

Nesse enfoque, ao pensar na estruturação de Programas de Transição de Cuidados, voltados para idosos hospitalizados, observou-se concordância entre os autores (Hinch et al., 2021; Poelzl et al., 2021; Gilbert et al., 2021; Crannage et al., 2020; Neu et al., 2020; Baecker et al., 2020; Vearing et al., 2019; Wu et al., 2019; Warren et al., 2019; Huckfeldt et al., 2019; Murphy et al., 2019; Finlayson et al., 2018; Ballard et al., 2018; Robertson et al., 2018; Hanan et al., 2018; Iseler et al., 2018; Low et al., 2017; Takahashi et al., 2016; Heim et al., 2016; Wong et al., 2016; Morrison et al., 2016; Lovelace et al., 2016; Zhou et al., 2015; Low et al., 2015; Mae et al., 2014; Wee et al., 2014; Cheng et al., 2014; Logue et al., 2013; Kind et al., 2012; Shu et al., 2011; Stauffer et al., 2011; Ornstein et al., 2011; Daley et al., 2010) acerca do impacto positivo desses programas, principalmente no que tange à redução das taxas de reinternação hospitalar em até 30 dias (Hinch \& Staffileno, 2021; Gilbert et al., 2021; Crannage et al., 2020; Wu et al., 2019; Warren et al., 2019; Huckfeldt et al., 2019; Low et al., 2017; Wong et al., 2016; Morrison et al., 2016; Zhou et al., 2015; Low et al., 2015; Ornstein et al., 2011; Daley et al., 2010) e melhoria da autonomia do idoso para condução de sua reabilitação (Hinch et al., 2021; Poelzl et al., 2021; Daliri et al., 2021; Baecker et al., 2020; Vearing et al., 2019; Wu et al., 2019; Warren et al., 2019; Huckfeldt et al., 2019; Ballard et al., 2018; Robertson et al., 2018; Hanan et al., 2018; Iseler et al., 2018; Takahashi et al., 2016; Heim et al., 2016; Wong et al., 2016; Morrison et al., 2016; Lovelace et al., 2016; Zhou et al., 2015; Low et al., 2015; Mae et al., 2014; Cheng et al., 2014; Logue et al., 2013; Kind et al., 2012; Shu et al., 2011; Stauffer et al., 2011), portanto é importante a implementação de ações em saúde estratégicas.

No que se refere às taxas de readmissão hospitalar, é concordância entre os autores que, em média, 20\% dos pacientes idosos que receberam alta hospitalar para domicílio experimentaram algum tipo de evento adverso evitável, sobre sua condição de saúde, que acarretou o processo de readmissão hospitalar. Dentre os quais, dois terços desses eventos estão relacionados ao manejo incorreto da terapêutica medicamentosa prescrita para uso em domicílio (Heim et al., 2016).

Nesse sentido, para Murphy et al. (2019), um componente essencial que deve estar presente em Programas de Transição de Cuidados, para lidar com o problema supracitado, é a adoção de ações educacionais durante o processo de 
Research, Society and Development, v. 11, n. 3, e35611326591, 2022

(CC BY 4.0) | ISSN 2525-3409 | DOI: http://dx.doi.org/10.33448/rsd-v11i3.26591

hospitalização, voltadas aos cuidados com a terapêutica medicamentosa usual de idosos. Essas ações devem enfocar, principalmente, no entendimento sobre a importância de adotar a medicação e as formas de evitar erros comuns na gestão dos medicamentos. Destaca-se que, para a determinação de um cuidado efetivo para essa parcela da população, é importante definir o idoso como intérprete do seu amparo, sendo necessário que ele tenha conhecimento da sua situação de saúde e seja estimulado a desenvolver autonomia diante da tomada de decisões (Murphy et al., 2019).

Para tanto, Neu e demais pesquisadores (2020), ao analisar um Programa de Transição de Cuidados para idosos que demonstrou eficácia significativa, no ponto de vista estatístico, na redução das taxas de reinternação hospitalar (10,5\% no grupo intervenção, contra $17,3 \%$ no grupo controle), propuseram três intervenções com o objetivo de combater problemas relacionados ao uso de medicamentos. São as seguintes: 1) revisão da terapêutica medicamentosa com um profissional da saúde - seja ele médico, enfermeiro ou farmacêutico - durante o processo de internação, abordando a função do fármaco, riscos de alergia e os efeitos adversos comuns e incomuns mediante o diagnóstico; 2) discussão envolvendo médicos e enfermeiros sobre o plano terapêutico proposto para adoção, em domicílio, durante o processo de alta hospitalar e quais as estratégias educacionais que pudessem ser usadas para efetivar o entendimento do paciente segundo as prescrições e 3) aconselhamento do paciente e/ou cuidador, por meio de materiais educacionais, com foco nos cuidados para uso de medicamentos.

No entanto, os impactos de redução nas taxas de readmissão hospitalar, com o uso somente de estratégias educativas em ambiente intra-hospitalar, não foram, estatisticamente, significativas para Donaho et al. (2015). Tal resultado pode estar relacionado à necessidade de elaborar ações em saúde no contexto pós-alta hospitalar, tendo em vista a eficácia observada nos demais estudos (Gilbert et al., 2021; Vearing et al., 2019; Wu et al., 2019; Huckfeldt et al., 2019; Murphy et al., 2019; Finlayson et al., 2018; Robertson et al., 2018; Low et al., 2017; Takahashi et al., 2016; Wong et al., 2016; Morrison et al., 2016; Lovelace et al., 2016; Shu et al., 2011), que utilizaram, de forma mista, ações educativas, de gerenciamento e supervisão de medicações, tanto em ambiente intra-hospitalar quanto em domicílio. Face ao exposto, uma estratégia predominante entre os autores para a supervisão e gestão de medicações, foi incluir a figura de profissionais multidisciplinares com a função de supervisionar o programa.

Segundo Crannage et al. (2020), a inserção do profissional farmacêutico no cuidado multidisciplinar torna-se relevante, a fim de garantir a eficácia de programas de transição do cuidado quanto à gestão de erros de medicação, por parte dos usuários. O farmacêutico é o profissional qualificado e apto a identificar e corrigir discrepâncias em receituários médicos, além de fornecer capacitação para o paciente e supervisão de sua terapêutica medicamentosa, impactando diretamente na redução da incidência de efeitos adversos evitáveis.

A figura do profissional enfermeiro demonstrou ser igualmente efetiva na supervisão de medicações, sendo descrita por autores (Gilbert, T. et al., 2021; Crannage et al., 2020; Neu et al., 2020; Warren et al., 2019; Murphy et al., 2019; Finlayson et al., 2018; Robertson et al., 2018; Hanan et al., 2018; Iseler et al., 2018; Low et al., 2017; Takahashi et al., 2016; Heim et al., 2016; Wong et al., 2016; Morrison et al., 2016; Lovelace et al., 2016; Zhou et al., 2015; Low et al., 2015; Mae et al., 2014; Wee et al., 2014; Cheng et al., 2014; Logue et al., 2013; Kind et al., 2012; Shu et al., 2011; Stauffer et al., 2011) como essencial no processo de comunicação entre os diferentes níveis da atenção à saúde, no processo de transição do cuidado do hospital para domicílio.

No que tange à qualificação desses profissionais, para cumprir a função de supervisão efetiva, Huckfeldt et al. (2019) defendem a estratégia do diálogo multidisciplinar com a equipe de Atenção Primária à Saúde. O objetivo é definir ações de acordo com as singularidades de cada idoso, de modo que ocorra a capacitação e promoção da autonomia dos pacientes, de modo a compreender suas fragilidades e predisposição à ocorrência de eventos que acometam seu estado de saúde.

No que diz respeito às ações em saúde aplicadas durante a internação, embora poucos estudos tenham implementado 
Research, Society and Development, v. 11, n. 3, e35611326591, 2022

(CC BY 4.0) | ISSN 2525-3409 | DOI: http://dx.doi.org/10.33448/rsd-v11i3.26591

intervenções terapêuticas no ambiente intra-hospitalar, observou-se concordância entre os autores, acerca do papel de planos de alta especializados, que se constituem como o alicerce de Programas de Transição de Cuidados para idosos (Gilbert et al., 2021; Deng et al., 2020; Murphy et al., 2019; Aboumatar et al., 2018; Ballard et al., 2018; Low et al., 2017; Takahashi et al., 2016; Heim et al., 2016; Zhou et al., 2015; Wong et al., 2015; Naylor et al., 2013; Stauffer et al., 2011). Vale destacar que, para Hanan et al. (2018), os planos de alta devem apresentar componentes como: orientações para paciente, familiares e/ou cuidador acerca do prognóstico e terapêutica medicamentosa e de reabilitação que será adotada em domicílio; agendamento de consulta de retorno, com média de até sete dias após alta hospitalar; orientações acerca de possíveis eventos adversos relacionados ao estado de saúde, orientações e encaminhamento para a equipe de Atenção Primária à Saúde sobre o caso.

Em estudo de Robertson e pesquisadores (2018), cujo programa de transição do cuidado iniciou ainda em ambiente hospitalar, foi possível concluir uma associação entre planos de alta especializados e uma redução das taxas de readmissão hospitalar, em 30 dias. Neste caso, o programa de alta consistiu em uma prescrição de cuidados intra-hospitalares para facilitar a adesão à medicação e consultas de alta prolongada, com uma enfermeira treinada para enfatizar os cuidados pós-operatórios, medicamentos, cuidados incisionais, nutrição, sinais que merecem retorno ao atendimento médico e agendamento de consultas de acompanhamento. Além disso, após a alta hospitalar, os pacientes receberam um telefonema de vigilância, decorridas 48 horas.

Essa ação de contato, após alta hospitalar, está presente em uma parcela da amostra de artigos eleita (Crannage et al., 2020; Finlayson et al., 2018; Robertson et al., 2018; Iseler et al., 2018; Low et al., 2015), constituindo-se também como uma ação essencial para efetivar Programas de Transição de Cuidados, sob o formato de acompanhamento telefônico ou visitas domiciliares.

Quanto ao tempo para implementar a ação, na visão de Baecker e colaboradores (2020), o tempo ideal para início é de dois dias após a alta hospitalar. Destaca-se que o tempo de duração das ações foi aplicado pela maioria dos autores, por um período de 30 dias, que também foi o tempo máximo de duração da maioria dos Programas de Transição de Cuidados descritos na amostra de artigos eleitos por esse estudo (Neu et al., 2020; Wu et al., 2019; Warren et al., 2019; Logue et al., 2013; Kind et al., 2012).

Para Vearing e colegas (2019), além de proporcionar a integralização do cuidado, tendo em vista a continuidade do cuidar no processo de transferência do idoso do hospital para domicílio, ações de visitas domiciliares são tidas como uma ponte para implementar diferentes ações em saúde, dentro de Programas de Transição de Cuidados, com vista, principalmente, para a reabilitação nutricional, física e psicológica do idoso.

Ainda no estudo de Vearing supracitado (2019), foi possível observar uma redução de $10 \%$ nas taxas de readmissão hospitalar em 30 dias, por meio de um Programa de Transição de Cuidados que consistiu em acompanhamento domiciliar pósalta hospitalar, por uma equipe multidisciplinar composta por nutricionistas, fisioterapeutas, terapeutas ocupacionais, fonoaudiólogos, enfermeiras e psicólogos. Os participantes receberam suporte dietético individualizado, dependendo de suas necessidades e requisitos, além de avaliação nutricional rotineira, por meio de instrumentos semiestruturados que foram aplicados durante as visitas domiciliares de terapeutas e nutricionistas. Foram evidenciadas melhorias autorreferidas pelos idosos acerca da saúde mental, referentes ao processo de adoecimento.

Por fim, observa-se que, para o amparo integralizado ao idoso, Programas de Transição de Cuidados se apresentam como uma ferramenta que deve ser utilizada para assegurar a assistência em saúde continuada, no processo de transferência dos pacientes entre os diferentes níveis de atenção à saúde. No entanto, faz-se necessário refletir que a base de Programas de Transição de Cuidados se dá por meio da comunicação entre os diferentes focos de atenção e, como já é sabido, sobre as dificuldades de interoperabilidade e compartilhamento de informações entre os níveis de atenção em saúde. Torna-se 
Research, Society and Development, v. 11, n. 3, e35611326591, 2022

(CC BY 4.0) | ISSN 2525-3409 | DOI: http://dx.doi.org/10.33448/rsd-v11i3.26591

imprescindível o papel de agentes regulamentadores que assegurem processos de transição, que contemplem a atenção integral e adoção das principais ações em saúde, previstas em Programas de Transição de Cuidados.

Embora os estudos elencados nesta revisão tenham apresentado contextos externos à realidade brasileira, o que dificulta a compreensão da eficácia de Programas de Transição de Cuidados para idosos no país, é válido dizer que, na lógica das Redes de Atenção à Saúde, o Brasil possui portabilidade para implementação desses programas, fazendo-se necessário adaptá-los para o Sistema Único de Saúde (SUS).

\section{Conclusão}

A revisão integrativa possibilitou observar a potencialidade de Programas de Transição de Cuidados para idosos, que impactam diretamente na melhora da adesão à medicação, do estilo de vida saudável e indicadores clínicos, bem como taxas menores de eventos adversos e readmissões hospitalares. O cuidado configura-se por meio de ações em saúde como: educação do paciente, familiares e/ou cuidador, ainda em ambiente hospitalar; acompanhamento telefônico e domiciliar após alta; supervisão e gestão de medicações e criação de planos terapêuticos individuais que visem a reabilitação nutricional, física e cognitiva do paciente. Recomenda-se a realização de estudos acerca de ações em saúde adotadas em outros contextos, após alta hospitalar, como em hospitais de retaguarda ou instituições de permanência para idosos, que possam contribuir diretamente para a estruturação de Programas de Transição de Cuidados, promovendo visibilidade de ações eficazes que são implementadas, mas ainda não evidenciaram sua relação com Programas de Transição de Cuidados para idosos.

\section{Referências}

Aboumatar, H., Naqibuddin, M., Chung, S., Chaudhry, H., Kim, S. W., Saunders, J., Bone, L., Gurses, A. P., Knowlton, A., Pronovost, P., Putcha, N., Rand, C., Roter, D., Sylvester, C., Thompson, C., Wolff, J. L., Hibbard, J. \& Wise, R. A. (2019). Effect of a Hospital-Initiated Program Combining Transitional Care and Long-term Self-management Support on Outcomes of Patients Hospitalized With Chronic Obstructive Pulmonary Disease: A Randomized Clinical Trial. Journal Of The American Medical Association, 322(14), 1371-1380.

Aboumatar, H., Naqibuddin, M., Chung, S., Chaudhry, H., Kim, S. W., Saunders, J., Bone, L., Gurses, A. P., Knowlton, A., Pronovost, P., Putcha, N., Rand, C., Roter, D., Sylvester, C., Thompson, C., Wolff, J. L., Hibbard, J. \& Wise, R. A. (2018). Effect of a Program Combining Transitional Care and Long-term Self-management Support on Outcomes of Hospitalized Patients With Chronic Obstructive Pulmonary Disease A Randomized Clinical Trial (Retracted article. See vol. 322, pg. 1417, 2019). Journal Of The American Medical Association, 320(22), 2335-2343.

Baecker, A., Meyers, M., Koyama, S., Taitano, M., Watson, H., Machado, M. \& Nguyen, H. Q. (2020). Evaluation of a Transitional Care Program After Hospitalization for Heart Failure in an Integrated Health Care System. Jama Network Open, 3(12).

Ballard, J., Rankin, W., Roper, K. L., Weatherford, S. \& Cardarelli, R. (2018). Effect of Ambulatory Transitional Care Management on 30-Day Readmission Rates. American journal of medical quality, 33(6), 583-589.

Ministério da Saúde (BR). (2013). Estratégias para o cuidado da pessoa com doença crônica: diabetes mellitus. Cadernos de Atenção Básica, n. 36. Ministério da Saúde. Brasília, p. 160.

Burke, R. E. Kripalani, S., Vasilevskis, E. E. \& Schnipper, J. L. (2013). Moving beyond readmission penalties: creating an ideal process to improve transitional care. Journal of Hospital Medicine, 8(2), 102-109.

Carret, M. L. V., Fassa, A. G., Paniz, V. M. V. \& Soares, P. C. (2011). Características da demanda do serviço de saúde de emergência no sul do Brasil. Ciência \& Saúde Coletiva, 16(1), 79-106.

Centeno, M. M. \& Kahveci, K. L. (2014). Transitional care models: Preventing readmissions for high-risk patient populations. Critical Care Nursing Clinics of North America, 26(4), 589-597.

Coleman, E. A. \& Boult, C. (2003). Improving the quality of transitional care for persons with complex care needs. Journal of the American Geriatrics Society, 51(4), 556-7, 2003.

Crannage, A. J., Hennessey, E. K., Challen, L. M., Stevens, A. M. \& Berry, T. M. (2020). Implementation of a Discharge Education Program to Improve Transitions of Care for Patients at High Risk of Medication Errors. Annals Of Pharmacotherapy, 54(6), 561-566.

Daliri, S., Kooij, M. J., Reimer, W. J. M. S. O., Riet, G., Jepma, P., Verweij, L., Peters, R. J. G., Buurman, B. M. \& Karapinar-Carkit, F. (2021). Effects of a transitional care programme on medication adherence in an older cardiac population: A randomized clinical trial. British Journal Of Clinical Pharmacology. 
Research, Society and Development, v. 11, n. 3, e35611326591, 2022

(CC BY 4.0) | ISSN 2525-3409 | DOI: htttp://dx.doi.org/10.33448/rsd-v11i3.26591

Daley, C. M. (2010). A hybrid transitional care program. Critical Pathways In Cardiology, 9(4), 231-234.

Deng, A., Yang, S. \& Xiong, R. (2020). Effects of an integrated transitional care program for stroke survivors living in a rural community: a randomized controlled trial. Clinical Rehabilitation, 34(4), 524-532.

Donaho, E. K., Hall, A. C., Gass, J. A., Elayda, M. A., Lee, V., Paire, S. \& Meyers, D. E. (2015). Protocol-Driven Allied Health Post-Discharge Transition Clinic to Reduce Hospital Readmissions in Heart Failure. Journal Of The American Heart Association, 4(12).

Finlayson, K., Chang, A. M., Courtney, M. D., Edwards, H. E., Parker, A. W., Hamilton, K. Y. R. A., Pham, T. D. X. \& O'Brien, J. (2018). Transitional care interventions reduce unplanned hospital readmissions in high-risk older adults. BMC health services research, 18(1), 956-956.

Garcia, A. K. A., Fonseca L. F., Aroni P. \& Galvão C. M. (2016). Strategies for thirst relief: integrative literature review. Revista Brasileira de Enfermagem, 69(6), 1148-55.

Goulart, F. A. A. (2011). Doenças Crônicas não Transmissíveis: Estratégias de controle e desafios para os sistemas de saúde. Organização Pan-Americana da Saúde, Ministério da Saúde. Brasília, p. 92.

Heim, N., Rolden, H., Van Fenema, E. M., Weverling-Rijnsburger, A.W.E., Tuijl, J. P., Jue, P., Oleksik, A. M., de Craen, A. J. M., Mooijaart, S. P., Blauw, G. J., Westendorp, R. G. J., Van Der Mast, R. C. \& Van Everdinck, I. E. C. (2016). The development, implementation and evaluation of a transitional care programme to improve outcomes of frail older patients after hospitalisation. Age Ageing, 45(5), 642-651.

Hinch, B. K. \& Staffileno, B. A. (2021). Implementing a Heart Failure Transition Program to Reduce 30-Day Readmissions. Journal for Healthcare Quality, $43(20,110-118$.

Huckfeldt, P. J., Reyes, B., Engstrom, G., Yang, Q., Diaz, S., Fahmy, S. \& Ouslander, J. G. (2019). Evaluation of a Multicomponent Care Transitions Program for High-Risk Hospitalized Older Adults. Journal of the American Geriatrics Society, 67(12), 2634-2642.

Iseler, J., Fox, J. \& Wierenga, K. (2018). Performance Improvement to Decrease Readmission Rates for Patients With a Left Ventricular Assist Device. Progress in transplantation, 28(2), 184-188.

Jepma, P., Verweij, L., Buurman, B. M., Terbraak, M. S., Daliri, S., Latour, C. H. M., Ter Riet, G., Karapinar-Çarkit, F., Dekker, J., Klunder, J. L., Liem, S. S., Moons, A. H. M., Peters, R. J. G. \& Scholte Op Reimer, W. J. M. (2021). The nurse-coordinated cardiac care bridge transitional care programme: a randomised clinical trial. Age and ageing, 50(6), 2105-2115.

Kind, A. J., Jensen, L., Barczi, S., Bridges, A., Kordahl, R., Smith, M. A. \& Asthana, S. (2012). Low-cost transitional care with nurse managers making mostly phone contact with patients cut rehospitalization at A VA hospital. Health Affairs, 31(12), 2659-2668.

Logue, M. D. \& Drago, J. (2013). Evaluation of a modified community based care transitions model to reduce costs and improve outcomes. BMC Geriatrics, $13(1)$.

Lovelace, D., Hancock D., Hughes S. S., Wyche P. R., Jenkins C. \& Logan, C. (2016). A Patient-Centered Transitional Care Case Management Program: Taking Case Management to the Streets and Beyond. Professional case management, 21(6), 277-290.

Low, L. L., Tan, S. Y., Ng, M. J. M., Tay, W. Y., Ng, L. B., Balasubramaniam, K., Towle, R. M. \& Lee, K. H. (2017). Applying the integrated practice unit concept to a modified virtual ward model of care for patients at highest risk of readmission: A randomized controlled trial. PLoS ONE, 2(1)

Low, L. L., Vasanwala, F. F., Ng, L. B., CHEN, C., Lee, K. H. \& Tan, S. Y. (2015). Effectiveness of a transitional home care program in reducing acute hospital utilization: A quasi-experimental study. BMC Health Services Research, 15(1).

Mendes, K. S., Silveira, R. C. C. P. \& Galvão, C. M. (2008). Revisão integrativa: método de pesquisa para a incorporação de evidências na saúde e na enfermagem. Texto \& Contexto - Enfermagem, 17(4), 758-764.

Melnyk, B. M. \& Fineout-Overholt, E. (2001). Evidence-based practice in nursing \& healthcare: a guide to best practice. Lippincott Williams \& Wilkins.

Morrison, J., Palumbo, M. V. \& Rambur, B. (2016). Reducing Preventable Hospitalizations With Two Models of Transitional Care. Journal of nursing scholarship, 48(3), 322-9.

Mourad, O., Hossam, H., Zbys F. \& Adn A. E. (2016). Rayyan - a web and mobile app for systematic reviews. Systematic Reviews, 5, 20.

Murphy, J. A., Schroeder, M. N., Rarus, R. E., Yakubu, I., Mckee, S. O. P. \& Martin, S. J. (2019). Implementation of a Cardiac Transitions of Care Pilot Program: A Prospective Study of Inpatient and Outpatient Clinical Pharmacy Services for Patients With Heart Failure Exacerbation or Acute Myocardial Infarction. Journal of Pharmacy Practice, 32(1), 68-76.

Naylor, M. D., Bowles, K. H., Mccauley, K. M., Maccoy, M. C., Maislin, G., Pauly, M. V. \& Krakauer, R. (2013). High-value transitional care: Translation of research into practice. Journal of Evaluation in Clinical Practice, 19(5), 727-733.

Neu, R., Leonard, M. A., Dehoorne, M. L., Scalia, S. J., Kale-Pradhan, P. B. \& Giuliano, C. A. (2020). Impact of Pharmacist Involvement in Heart Failure Transition of Care. Annals of Pharmacotherapy, 54(3),239-246.

Ornstein, K.,Smith, K. L., Foer D. H., Lopez-Cantor, M. T. \& Soriano, T. T. (2011). The hospital and back home again: a nurse practitioner-based transitional care program for hospitalized homebound people. Journal of the American Geriatrics Society, 59(3), 544-51.

Poelzl, G., Egelseer-Bruendl, T., Pfeifer, B., Modre-Osprian, R., Welte, S., Fetz, B., Krestan, S., Haselwanter, B., Zaruba, M. M., Doerler, J., Rissbacher, C., Ammenwerth, E. \& Bauer, A. (2021). Feasibility and effectiveness of a multidimensional post-discharge disease management programme for heart failure patients in clinical practice: the HerzMobil Tirol programme. Clinical Research In Cardiology. 
Research, Society and Development, v. 11, n. 3, e35611326591, 2022

(CC BY 4.0) | ISSN 2525-3409 | DOI: htttp://dx.doi.org/10.33448/rsd-v11i3.26591

Registered Nurses’ Association Of Ontario (2014). Care transitions. Clinical best practice guidelines. Toronto: RNAO.

Robertson, F. C, Logsdon, J. L., Dasenbrock, H. H., Yan, S. C., Raftery, S. M., Smith, T. R. \& Gormley, W. B. (2018). Transitional care services: a quality and safety process improvement program in neurosurgery. Journal of neurosurgery, 128(5), 1570-1577.

Robinson, T. E., Zhou, L., Kerse, N., Scott, J. D. R., Christiansen, J. P., Holland, K., Armstrong, D. E. \& Bramley, D. (2015). Evaluation of a New Zealand program to improve transition of care for older high risk adults. Australasian Journal on Ageing, 34(4), 269-274.

Rossetto, C., Soares J. V., Brandão, M. L., Rosa, N. G. \& Rosset, I. (2019). Causas de internação hospitalar e óbito em idosos brasileiros entre 2005 e 2015. Revista Gaúcha de Enfermagem,40, e20190201.

Shimidt, M. I. (2011). Chronic non-communicable diseases in Brazil: burden and current challenges. The Lancet, 377(9781), 1949-1961.

Stauffer, B. D., Fullerton, C., Fleming, N., Ogola, G., Herrin, J., Stafford, P. M. \& Ballard, D. J. (2011). Effectiveness and Cost of a Transitional Care Program for Heart Failure A Prospective Study With Concurrent Controls. Archives Of Internal Medicine, 171(14), $1238-1243$.

Shu, C., Hsu, N., Lin, Y., Wang, J., Lin, J. K. \& Wen J.E. (2011). Integrated postdischarge transitional care in a hospitalist system to improve discharge outcome: an experimental study. BMC Medicine, 9.

Takahashi, P. Y., Naessens, J. M., Peterson, S. M., Rahman, P. A., Shah, N. D., Finnie, D. M., Weymiller, A. J., Thorsteinsdottir, B. \& Hanson, G. J. (2016). Short-term and long-term effectiveness of a post-hospital care transitions program in an older, medically complex population. Healthcare, 4(1), 30-35.

Vearing, R., Casey, S., Zaremba, C., Bowden, S., Ferguson, A., Goodisson, C., Potter, J., Evry, N. \& Charlton, K. (2019). Evaluation of the impact of a posthospital discharge Transitional Aged Care Service on frailty, malnutrition and functional ability. Nutrition and Dietetics, 76(4), 472-479.

Warren, C., Lemieux, A. A. \& Bittner, N. P. (2019). Excellence in Population Health A Successful Community-Based Care Transitions Program Model. Professional Case Management, 24(1), 39-45.

Wee, S., Loke, C., Liang, C., Ganesan, G., Wong, L. \& Cheah, J. (2014). Effectiveness of a National Transitional Care Program in Reducing Acute Care Use. Journal Of The American Geriatrics Society, 62(4), 747-753.

Wong, F. K. Y. \& Yeung, S. M. (2015). Effects of a 4-week transitional care programme for discharged stroke survivors in Hong Kong: A randomised controlled trial. Health and Social Care in the Community, 23(6), 619-631.

Wong F.K., Ng A., Lee P.H., Lam P.T., Ng J.S., Ng N.H. \& Sham M.M. (2016). Effects of a transitional palliative care model on patients with end-stage heart failure: a randomised controlled trial. Heart (British Cardiac Society), 102(14), 1100-1108.

Wong A. K. C., Wong F. K. Y., Ngai J. S. C., Hung S. Y. K. \& Li W. C. (2020). Effectiveness of a health-social partnership program for discharged non-frail older adults: a pilot study. BMC geriatrics, $20(1), 339$.

Wu, Q., Zhang, D., Zhao, Q., Liu, L., He, Z., Chen, Y., Huang, H., Hou, Y., Yang, X. \& Gu, J. (2019). Effects of transitional health management on adherence and prognosis in elderly patients with acute myocardial infarction in percutaneous coronary intervention: A cluster randomized controlled trial. $P L O S O N E$, $14(5)$. 